\title{
Analysis of Reasons and Social Benefit for the Charge Dispute between Telecom Operators and WeChat
}

\author{
Meijuan $\mathrm{Li}^{\mathrm{a}}$, Lei Hou ${ }^{\mathrm{b}}$ \\ ${ }^{\text {a }}$ School of Economics and Management, Yunnan Normal University \\ Kunming and 650500, China \\ ${ }^{\mathrm{b}}$ School of Economics and Management, Yunnan Normal University \\ Kunming and 650500, China
}

\begin{abstract}
Based on the charge disput between Chinese telecom operators and WeChat, this paper analyzes the reasons for the dispute by sorting out the event. Firstly, the transformation of the mobile Internet business model give rise to the marginalization of market interest entities as well as operators, which inevitably leads to the occurrence of disputes. Secondly, the rapid growth of the demand for mobile Internet services has promoted the maintenance and investment of base stations by telecom operators, which has increased the operators' operating cost pressures. Lastly, the technical reason. When WeChat sends a large amount of signaling, this inevitably causes interference to the mobile operator's call quality. From the perspective of network neutrality, WeChat charges will reduce the profits of content providers, which will result in a decrease in the effective number of content providers in the market, and is not conducive to the development of content providers and the utility of mobile phone users. Although the charging on WeChat will bring additional access fee for the telecom operators, it may not necessarily bring positive effects to the overall social development.
\end{abstract}

Keywords-Telecom operators; Charging on WeChat; Mobile internet; Network neutrality

\section{INTRODUCTION}

In January 2011, Tencent launched a service program that mainly provides instant messaging applications for smart terminals. It was named "WeChat." The initial positioning of the product itself is completely free to use for users. Users only need to pay network fees to the telecom operator, and they can enjoy all the services of the product. WeChat can transmit text, voice, pictures, video, and other content sections through the mobile Internet, and support cross-platform and crossoperating system information transmission between different telecom operators. Since the launch of WeChat, it has been very popular among consumers. The relevant data shows that the number of WeChat users has reached 300 million in just two years after launch. The explosive growth of WeChat has attracted much attention from mobile operators. Especially in 2012, the 4.2 version of WeChat began to provide services for voice calls and video calls. These functions are considered to be strong competitors after the SMS and MMS functions to mobile operators. Under the impact of WeChat, operators' own business has become increasingly declining. In 2012, the nation's total volume of text messages was 897.31 billion, an increase of only $2.1 \%$ year-on-year, far below the $7.1 \%$ increase in 2010 and the $6.2 \%$ increase in 2011.

\section{Charge Dispute BetweEn TELECOM Operators AND WECHAT}

In December 2012, Li Yue, president of mobile communications company, publicly accused WeChat, claiming that the traditional services of telecommunication operators were severely impacted by OTT (Over The Top) Companies, making telecommunications operators' original SMS and voice call services face great Challenges, especially WeChat. There will be a detrimental impact on the quality of operators' communications services due to a large amount of data flow used by what that consumes a large number of signaling resources. Lu Yimin, general manager of China Unicom, said that the rapid development of WeChat had brought great challenges to telecom operators. At the beginning of 2013, the second "Lingnan Forum" was held. At the meeting, the Ministry of Industry and Information Technology (MIIT) convened three major operators and some OTT companies to discuss the impact of OTT companies on telecommunication operators. Miao Wei, Minister of the Ministry of Industry and Information Technology Speaking at the seminar, said: WeChat may be charged in the future, but the fee will not be too high. The WeChat team announced in April 2013 that it has started to work with telecommunication operators to study the solution to signaling problems. In April 2013, the spokesperson of the Ministry of Industry and Information Technology and the Director of Communications Development Zhang Feng stated in a regular press release: Whether it will be necessary to charge mobile Internet new services should be decided by the market. This is the first public statement of the WeChat charging event by the Ministry of Industry and Information Technology. Therefore, the dispute over WeChat fees was launched. 


\section{REASONS FOR THE CHARGE DISPUTE}

\section{A. The change of mobile internet business model}

With the advancement of mobile device technology, the mobile internet industry chain structure has undergone profound changes in recent years, and the previous business model has begun to change. The traditional services of telecom operators are subject to competition from the content providers. Some content provider services have an alternative effect on telecom operators' businesses, which directly weakens the telecom operators' original product market share.

With the popularization and application of smartphones, people's consumption habits have undergone tremendous changes. More and more mobile applications have come into people's sights. Content providers have gradually dominated the mobile internet market. Telecom operators gradually become data transmission channels [1]. The huge profits in the industry are divided by content providers. Operators can only rely on network flow service for relatively small profits. On the other hand, there are many similarities between the products provided by some content providers and their products, and there are strong alternatives. For example, Weibo, WeChat, WhatsUp, SKYPE and other new content products make people no longer restricted in short messages and calls to communicate. As a result, the original customer of the operator is preempted by the content provider, and profits are divided. In general, the change of the Internet business model has caused content providers to become the main interests of the market [2]. If operators are marginalized, it will inevitably lead to disputes.

\section{B. Increasing investment pressure of the Telecom operators}

Although the huge user demand for content providers directly brings huge network flow consumption for operators, the large amount of data flow brought by large-scale Internet companies (such as Google, Amazon, Alibaba, etc.) has aggravated the problem of network congestion, making the operation a higher maintenance and investment cost of the base station, and the network flow income does not bring substantial profits to the operators compared to the higher network investment costs [3].

With the development of $3 \mathrm{G}$ networks in 2013, the speed of mobile networks has been greatly improved, and mobile Internet network flow has begun to show explosive growth. According to an annual report released by Cisco, the proportion of Internet flow in 2010 was $97 \%$, and by the end of 2015, it had dropped to $87 \%$. The decrease in the proportion of PC-side flow indicates that the mobile Internet is occupying the market at a faster rate.

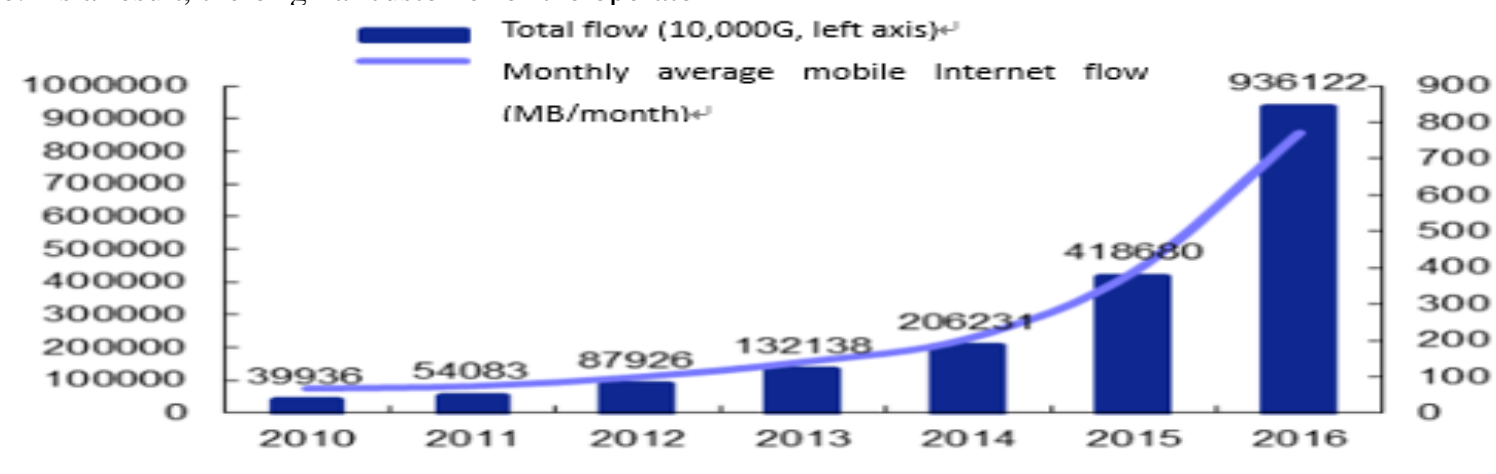

Fig. 1. Statistics on China's mobile Internet access flow from 2010 to 2016 (10,000 G)

a. Source: Ministry of Industry and Information Technology: "Statistical Bulletin of Communications Operations Industry 2016"

As can be seen from Figure 1, China's mobile Internet is growing at a very rapid rate in recent years, from 399 million in 2010 to 9.361 billion in 2016, an increase of 23.5 times in just six years. Among them, during the period from 2012 to 2016, the trend of explosive growth of network flow, especially during the period 2013 to 2014, the total mobile data flow of the three major operators increased by about $83 \%$. This is mainly due to the popularization of $3 \mathrm{G} / 4 \mathrm{G}$, which greatly accelerated the speed of surfing the Internet with the mobile terminals. Internet users gradually began to use mobile phones instead of computers to achieve some of the video requirements, which directly led to a surge in the mobile data flow. Also, the average monthly access flow of mobile phone users has rapidly increased from less than 100MB in 2010 to about $800 \mathrm{MB}$ in 2016. The huge traffic demand has brought many challenges to the operator platform and promoted the maintenance of base stations by operators. These maintenance and investment have increased pressure on operators' operating costs [4]. 


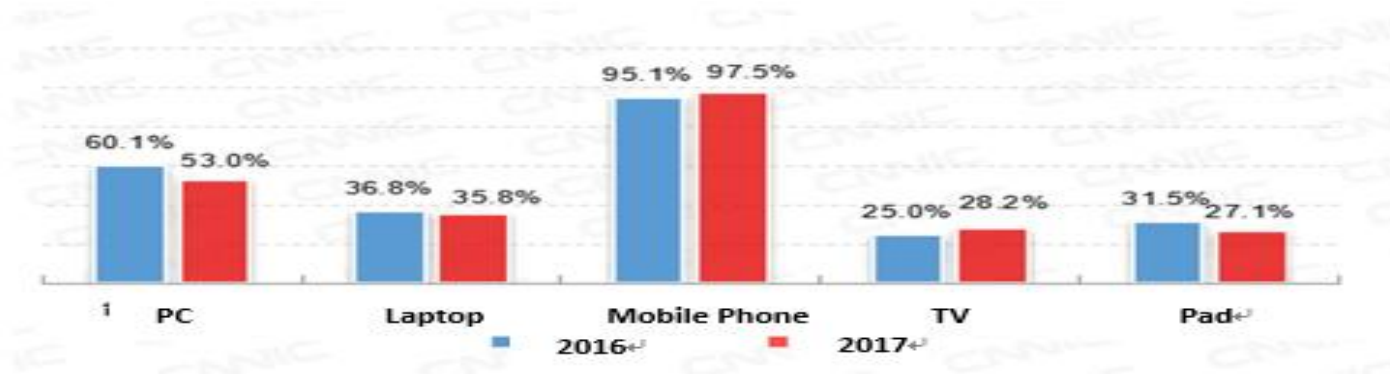

Fig. 2. Internet access equipment

b. Source: Ministry of Industry and Information Technology: "Communications and Operations Statistics Communique 2017"

As can be seen from Figure 2, the current mobile phone has become the main access device for the Internet. In 2017, the proportion of citizens using mobile phones to access the

Internet has reached $97.5 \%$, and the proportion of consumers using mobile phones to access the Internet continues to climb.

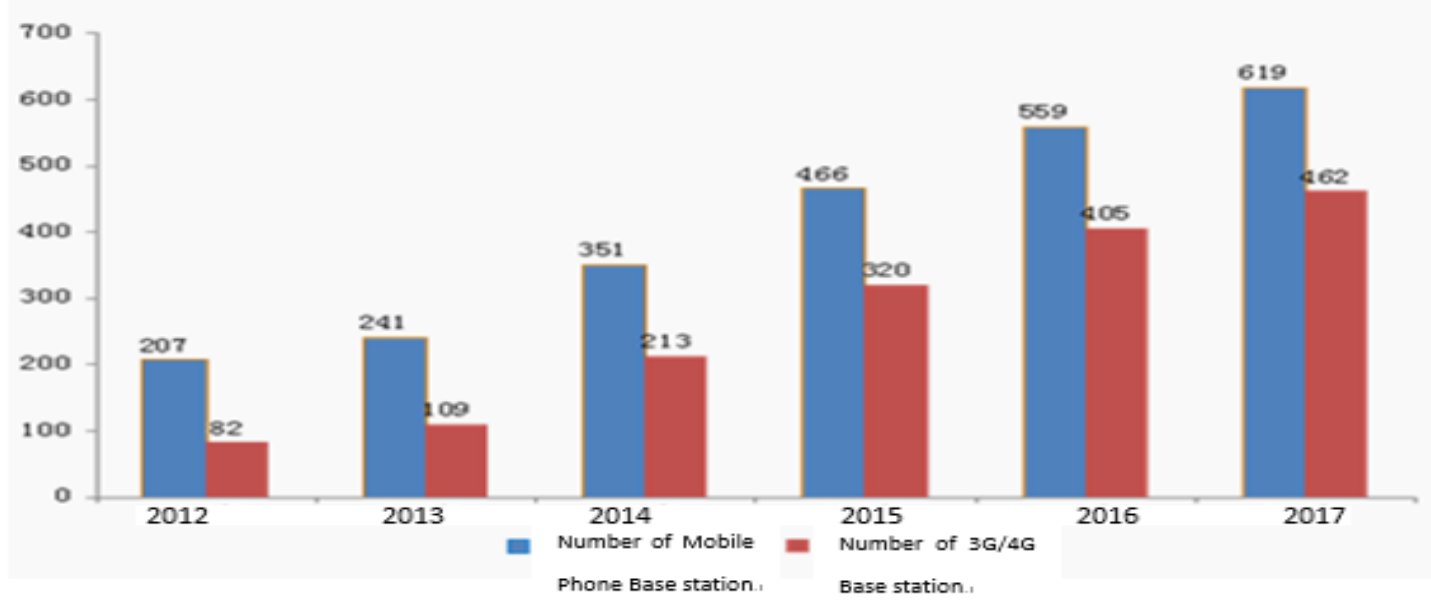

Fig. 3. Development of Mobile Phone Base Stations, 2012-2017 (Units Ten Thousand)

As can be seen from Figure 3, the number of $3 \mathrm{G} / 4 \mathrm{G}$ base stations in the mobile Internet market increased from 820,000 in 2012 to 4.62 million in 2017, an increase of 5 times. It is noteworthy that the growth rate in 2013 to 2014 is $95 \%$. From this, it can be seen that the operator base station investment is increasing. Given this, operators began to discuss charging content providers to make up for the huge investment costs.

\section{Technical reasons}

At the beginning of the launch of WeChat, due to the special characteristic of its product technology, it was necessary to frequently issue "heartbeat signals" to the operator platform [5]. This may occupy part of the signaling resources, affect the quality of wireless calls, and even affect the security of communications. The data shows that WeChat accounts for $60 \%$ of China Mobile's signaling, but it only generates $10 \%$ of the network flow. For this reason, the WeChat "charges" controversy, China Mobile's performance is the most positive.

Although China Mobile has already built the world's largest $2.5 \mathrm{G}$ network before 2013, it is technically lagging behind its competitors due to technical backwardness and comparison with more advanced $3 \mathrm{G}$ services abroad. A significant feature of the GSM network at that time is that the of Industry and Information Technology: "Communications and Operations Statistics Communique 2017" mobile phone users can not receive SMS at the same time when they answered the phone. Since the transmission of WeChat is the transmission of a "heartbeat signal," that is the signaling of the transmission information for the mobile operator. For telecom operators, signaling is mainly used to control voice call services. When WeChat sends a large amount of signaling, it will inevitably cause interference to the mobile operator's call quality. It is precisely because of this reason that it has led operators to "boycott" WeChat [6].

\section{The Wechat Challenge to Telecom Operators: TAKING CHINA MobILE AS AN EXAMPLE}

As early as 2009, China Mobile already had its similar products. Moreover, the birth of WeChat can be seen as a challenge for China Mobile.

Feixin can achieve mass distribution among mobile phone users. It can be described as an attempt by China Mobile to extend the downstream of the industry chain. However, as compared to WeChat, the attraction of Feixin products to users is greatly weakened. Therefore, WeChat can be considered as an extension to Feixin,an alternative product. The following figure shows the characteristics of WeChat and Feixin: 
TABLE I.

COMPARISON OF WeChat AND FEIXIN Product ATtRIBUTES

\begin{tabular}{c|c|c}
\hline \hline Product attributes & WeChat & Feixin \\
\hline Text messages & & \\
\hline Voice messages & & \\
\hline Photo Transfer & & \\
\hline video call & & \\
\hline polylogue & & \\
\hline Music Sharing & & \\
\hline Circle of Friends & & \\
\hline
\end{tabular}

As can be seen from Table 1, WeChat is complete than Feixin, not only has a unique business model such as text, voice, and video ,but also has functions like music sharing and friend circles which Feixin do not have. Because of this, users are more willing to choose WeChat as a communication tool.

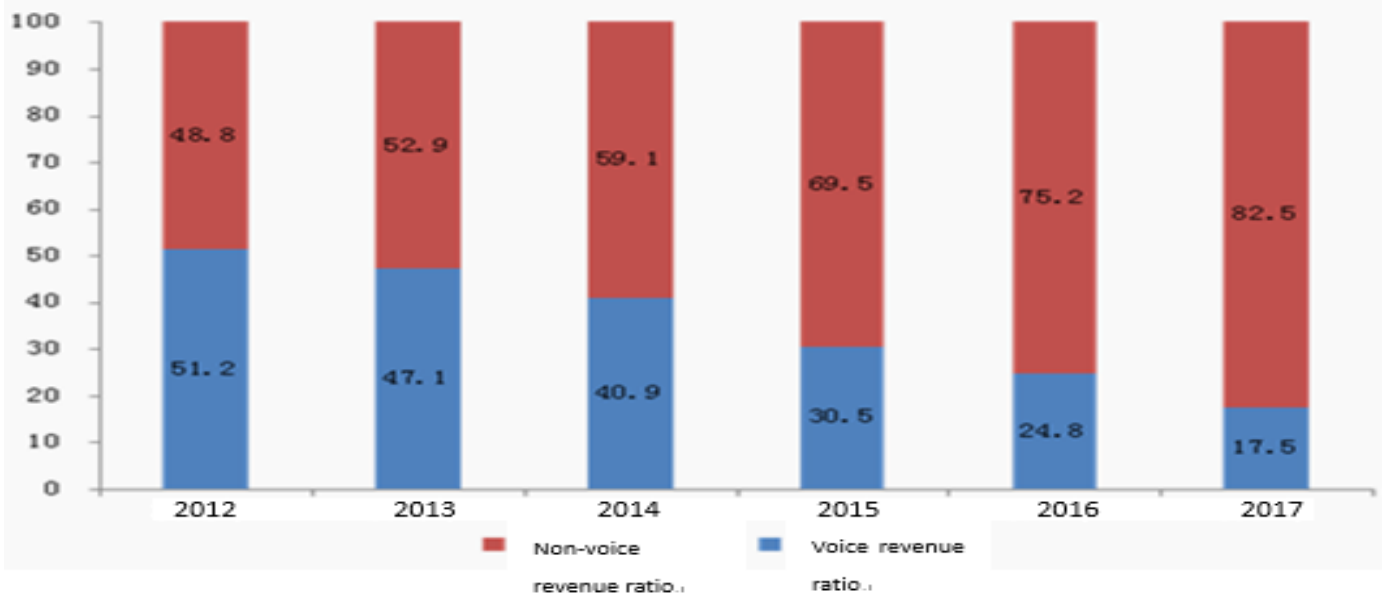

Fig. 4. Revenues of Telecom Operators

d. Source: Ministry of Industry and Information Technology: "Communications and Operations Statistics Communique 2017"

From Figure 4, it can be seen that since 2012, telecom operators' voice service revenue has begun to show a downward trend, and the proportion of surplus audio service revenue decreased from 51.2 in 2012 to 17.5 in 2017. This shows that more and more content providers with communication capabilities continue to erode the profits of telecom operators' own business, which inevitably causes the outbreak of disputes between the two.

\section{THE ANALYSIS OF SOCIAL WELFARE FOR WECHAT CHARGING}

Based on the above analysis, there are two possible ways for telecom operators to charge WeChat: one is to collect a one-time access fee, and the other is to charge according to flow usage. Regardless of the type of fee collection, this is unfavorable to Tencent as a content provider[7]. When telecom operators charge content providers such as Tencent, WeChat, and others, they will reduce the profits of content providers, and the industry's barriers to entry will also increase, which will affect the enthusiasm of content providers to enter the market. The decrease in the number will, in turn, affect the utility level of mobile phone users. Although the profits of telecom operators will increase, the overall social welfare may be reduced. Since WeChat has already shouldered the cost of data flow from some telecom operators, the price of telecom operators' charges for consumer-side data services will be reduced,consumers will receive more consumer surplus. At the same time, as profits are reduced, it is not conducive to the development of content providers represented by WeChat. It can thus be seen that China Mobile's charging of WeChat may have a positive effect and may also have a negative effect.

From a network neutral perspective, depending on the types of services provided by content providers, content providers have complementary and alternative effects to the traditional services of telecommunications operators. The replacement or complementarity effect in the business area is determined by the service attributes provided by the content provider. During the period of business growth, complementary and alternative effect will bring greater profits to operators and reduce their profits. When the benefits of complementary content providers exceed those of alternative content providers, content providers and operators can achieve mutually beneficial and win-win goals[8]. At the level of data services and traditional services, WeChat is complementary (WeChat can bring network flow consumption for telecom operators) and alternatives. The market impact caused by its substitution is far beyond complementarity, which has caused disputes. When the competitive relationship between telecom operators and content providers is dominated by substitution, the competitive relationship between the two will lead to a non-neutral network 
in the mobile Internet industry. All in all, WeChat charging will reduce the profits of content providers, which in turn will result in a decrease in the effective number of content providers in the market, which is not conducive to the development of content providers and the use of mobile phone users. Although WeChat charging will bring additional access fee income to the telecom operators, it may not necessarily bring positive effects to the overall social welfare.

\section{CONCLUSION}

Based on incidents in which Chinese telecom operators tried to charge fees from WeChat,we analyze the reasons for the charge dispute between telecom operators and WeChat,and the impact of social welfare on the mobile Internet market. The analysis results show that the shift in the business model of mobile Internet is the main reason for the emergence of charge disputes. When the alternative effect of the content provider's own business to mobile operators is more than complementary effect, it may have a negative impact on operators. As a result, telecom operators may adopt measures such as discriminatory pricing or impeding their access for content providers that occupy too much network resources. This will adversely affect for the healthy development of content providers, although it brings access revenue benefits for mobile operations, but the impact on total social welfare may be unfavorable.

\section{REFERENCES}

[1] Xu Xin. Can the Principle of Network Neutrality Solve the WeChat Charge Dispute? [J]. China Electric Power, 2014(07):64-67.

[2] Wang Hui, Fan Cunhuan. WeChat Charging, Total Social Welfare and Government Regulation [J]. Special Zone Economy, 2013(11): 200-202.

[3] Xu Zhaochang. Dispute on WeChat Charging: Analysis of Perspectives and Problems [J]. Journal of Guangzhou City Vocational College, 2013 (02): 18-22.

[4] Zheng Shurong. Opportunities for operators to cooperate and innovate in the dispute of WeChat charging [J]. C-Enterprise Management, 2014(02):62-63.

[5] Zhang JiChu. The bottleneck monopoly of anti-monopoly regulations from the dispute of WeChat charging [J]. Journal of the Shijiazhuang University of Economics, 2013(06):133-136.

[6] Lu Weigang. User heterogeneity, non-neutral network, and public policy: based on the perspective of the bilateral market [J]. China Industrial Economy, 2013, (02): 113-117.

[7] Chen Fuliang, Wang Lin. Research on Cooperation Strategies between Content Providers and Service Providers under non-neutral network [J]. Industrial Economics, 2013, (02): 45-50.

[8] Nie Jiajia, Li Weichen, Shi Chunlai. Research on cooperation strategy of content provider and service provider under non-neutral network [J]. Soft Science, 2017, (03): 125-130. 